\title{
Optical tweezers applied to a microfluidic system
}

\author{
Jonas Enger, Mattias Goksör, Kerstin Ramser, Petter Hagberg and Dag Hanstorp* \\ Department of Physics, Chalmers University of Technology and Göteborg University, SE-412 96 \\ Göteborg, Sweden. E-mail: dag.hanstorp@fy.chalmers.se; Fax: +46 31 7723496; Tel: +46 31 \\ 7723274
}

Received 11th July 2003, Accepted 18th February 2004

First published as an Advance Article on the web 19th March 2004

\begin{abstract}
We will demonstrate how optical tweezers can be combined with a microfluidic system to create a versatile microlaboratory. Cells are moved between reservoirs filled with different media by means of optical tweezers. We show that the cells, on a timescale of a few seconds, can be moved from one reservoir to another without the media being dragged along with them. The system is demonstrated with an experiment where we expose $E$. coli bacteria to different fluorescent markers. We will also discuss how the system can be used as an advanced cell sorter. It can favorably be used to sort out a small fraction of cells from a large population, in particular when advanced microscopic techniques are required to distinguish various cells. Patterns of channels and reservoirs were generated in a computer and transferred to a mask using either a sophisticated electron beam technique or a standard laser printer. Lithographic methods were applied to create microchannels in rubber silicon (PDMS). Media were transported in the channels using electroosmotic flow. The optical system consisted of a combined confocal and epi-fluorescence microscope, dual optical tweezers and a laser scalpel.
\end{abstract}

\section{Introduction}

Optical tweezers are a well-known technique for manipulation of micrometer-sized objects in a solution, as first shown by Arthur Ashkin. ${ }^{1}$ Individual cells are trapped in the focal point of a laser beam that is focused by a high NA microscope objective. By selecting a non-absorbing wavelength it is possible to trap almost any type of cell without causing harm. Optical tweezers are now widely used ${ }^{2}$ and they are particularly powerful in the field of microbiology. ${ }^{3-5}$ They have, for instance, been used to study cellcell interaction, ${ }^{6}$ to manipulate organelles without breaking the cell membrane ${ }^{7}$ and to measure adhesion forces between cells. ${ }^{8}$ It is even possible to drill holes in cell membranes at very precise positions using a so-called laser scalpel. ${ }^{9}$ One of the most obvious applications of optical tweezers is as a sorting device. ${ }^{10}$ This was shown by Ericsson et al. ${ }^{11}$ who studied bacterial viability. The optical tweezers were used to position bacteria in patterns on a microscope slide, where they were immobilized by the adhesion forces between the cell membrane and the glass surface. Another example is the work of Grover et al. who showed how optical tweezers can be applied in automated single cell-sorting using peripheral blood as a model system. ${ }^{12}$ The erythrocytes were aligned into an upright position along the beam propagation. Trapped cells could then be recognized and sorted out by an imageprocessing system. This automated single cell sorter is applicable when the sample consists of cells having non-spherical shapes or when an inhomogeneous cell population of varying size and shape is investigated. In general, experimental studies of cells using optical tweezers can reveal very interesting information, but the experiments are often time consuming and limited to cases where a small number of cells are investigated.

An alternative approach to control cells under the microscope is to produce microfluidic systems to transport, manipulate, sort and isolate different cells, ${ }^{13-15}$ also referred to as lab-on-a-chip devices. ${ }^{16-19}$ In such a microfluidic system, pressure or electroosmotic flow can be used to control the transport of the cells together with their surrounding media. ${ }^{20-22}$ These shrinking laboratories have allowed smaller and smaller samples to be used. A potential limitation, however, is the difficulty in changing the media around the cells under investigation without also losing control of the cells. Further, it is difficult to temporarily or permanently place a specific cell in a desired part of the chip. These systems are particularly powerful when there is an excess of identical cells available, which can be spread over the system where they interact with different probes. Recently, Umehara et al. ${ }^{23}$ and Inoue et al. ${ }^{24}$ improved their applicability by integrating optical tweezers into a lab-on-a-chip. They used the optical tweezers to position bacteria in a single-cell microcultivation assay where single bacterial cells could be isolated and positioned in microchambers. Subsequently, they were able to study the reproducibility of genetically identical bacteria by flowing different nutrients through the microchamber system. The nutrition was introduced to the individual chambers from a large reservoir through semipermeable membrane lids. This has the effect that the individual chambers were all exposed to the same media.

In this paper we show how the combination of optical tweezers and a lab-on-a-chip can be used to obtain full control of both a cell and its environment. First, we will demonstrate how cells can be positioned into compartments of a chip where a specific medium is present, or where further investigations can take place. In this case the single optically trapped cell is moved between the different reservoirs and it is shown that none of the different media are dragged along with the sample. Hence, contamination of different chambers and channels can be avoided, which opens up opportunities for a whole new series of interesting experiments. For instance, subsequent dynamics triggered by environmental stimuli can be observed in real time and valuable information such as direct drug response or stress can be studied. Alternatively, it is possible to hold a cell fixed and change its surrounding media by flowing different buffers through the channels using either an electro-osmotic flow or pressure variations. We will also demonstrate microsurgery on a cell in a specific medium, which subsequently is moved to another compartment, all performed with no mechanical contact and hence in a sterile environment. In addition, we will show that the system can be used as a cell sorter, where the transport is mainly performed by means of the flowing media in the channels. The selection of a specific cell and its guidance into a specific channel is performed with the optical tweezers. Cells can be injected and extracted from the system using micromanipulators. This method can be developed into an alternative method to conventional sorting techniques, such as fluorescence activated cell sorters (FACS). ${ }^{25-27}$ Since the lab-on-a-chip is easily custom tailored and manufactured for a 
series of varying experiments we believe that it has high potential within many microbiological or biomedical applications.

\section{Method}

In this section, the experimental techniques will be presented. We will describe how it is possible to simultaneously observe and manipulate particles in tailor-made environments. The optical tweezers can be used to trap any particle of interest and move it into different parts of the microfluidic system, independent of the flow direction of the surrounding media. Furthermore, the system is equipped with a laser scalpel consisting of a pulsed UV laser, which can be employed for carrying out microsurgery on single cells. In this study, the laser scalpel will be used to cut a hole in the membrane of Escherichia coli (E. coli) and the subsequent diffusion of propidium iodide will be monitored. In a previous study we have shown that this can be used to reveal unknown intracellular structures in mutants of $E$. coli bacteria. ${ }^{28}$ The optical parts of this system i.e., the microscope, the optical tweezers, and the laser scalpel have been described in detail elsewhere, ${ }^{29}$ and only a brief description is given here. The optical design of the optical tweezers essentially follows the design by Fällman et al. ${ }^{30}$ The microchannel system, on the other hand, is described in some more detail.

\section{The microscope}

All imaging in this work was performed with a Nikon TE300 microscope equipped either with an epi-fluorescence excitation system consisting of a $100 \mathrm{~W}$ mercury lamp for excitation and a CCD camera for detection, or a BioRad MRC1024 confocal scanning unit using an $\mathrm{Ar}-\mathrm{Kr}$ laser (488, 568 and $647 \mathrm{~nm})$. The objective used was an oil immersion Nikon IR 100X with a numerical aperture of 1.3. The microscope was also equipped with an Eppendorf micromanipulator system placed on a motorized microscope table, which was used for injection and extraction of the samples under observation.

\section{The optical tweezers}

Optical tweezers are constructed by directing a laser beam through the objective of the microscope. Efficient trapping is obtained if the laser beam completely fills up the entrance aperture of an objective with a high numerical aperture. ${ }^{4,30}$ An overfilled entrance aperture ensures stable trapping, where small variations in the laser beam direction has a negligible effect on the trapping efficiency. In this setup, shown in Fig. 1, we used a diode pumped Nd:YVO (1064 $\mathrm{nm})$ or a tunable Argon ion pumped titanium-sapphire laser $(780-850 \mathrm{~nm})$, for the optical tweezers. For this study a wavelength of $820 \mathrm{~nm}$ was used from the titanium-sapphire laser. ${ }^{31}$ To have good trapping these lasers delivered typically $30-90 \mathrm{~mW}$ of laser radiation measured before the objective. The laser beam was expanded by lenses $L_{1}$ and $L_{2}$ in order to overfill the entrance aperture of the objective. To get dual trap optical tweezers the laser beam was split into two arms using a polarizing beam splitting cube $\left(\mathrm{PBSC}_{1}\right)$. For separately moving the two optical tweezers freely within the specimen plane ( $x-y$ direction) in the microscope, each arm has a gimbal-mounted mirror (GMM). A second beam splitting cube $\left(\mathrm{PBSC}_{2}\right)$ is used to merge the two optical tweezers arms prior to the entrance of the microscope. By moving lens $\mathrm{L}_{3}$ or $\mathrm{L}_{4}$ in the direction of the propagation of the laser beam, the tweezers focus could be adjusted in the $z$-direction. This made it possible to overlap the trapping plane with the focal plane of the microscope. Just before the objective a filter cube with a dichroic mirror $\left(\mathrm{DM}_{2}\right)$ was used to reflect laser light as well as excitation light through the objective. The dichroic mirror was transparent for the fluorescence signal.

\section{The laser scalpel}

The optical scalpel is formed using a pulsed nitrogen laser with a wavelength of $337 \mathrm{~nm}$ and a pulse width of $4 \mathrm{~ns}$ (FWHM). The laser pulse is directed in the same path as the laser tweezers beam using a third dichroic mirror, $\left(\mathrm{DM}_{3}\right)$. The energy of the laser was reduced by neutral density filters (ND) to an energy of $0.6 \mu \mathrm{J}$ measured before the microscope objective.

\section{Manufacturing of a channel system}

We produced our microchannels either in an advanced clean room facility using an electron beam lithography, or in a standard chemical laboratory using a high quality laser printer. The electron beam method gives the highest resolution, but for channels with widths of the order of $20 \mu \mathrm{m}$ a standard laser printer is sufficient. Since the latter method can be performed in almost any laboratory, we present both methods here.

\section{In a cleanroom using electron beam}

The cell sorter was produced by poly(dimethyl siloxane) (PDMS) on a photoresist mask. The channel system layout was first designed in an ordinary CAD-program. The pattern was thereafter transferred to a mask on a chromium $(\mathrm{Cr})$-covered silicon glass plate using high resolution electron beam lithography (JBX-5DII, JEOL, Japan). This mask was used to create a negative relief of the structures according to the following procedure. A negative photoresist (SU-85, Micro Chem, USA) was spin-coated on a silicon wafer. Then, the substrate was soft-baked on a contact hotplate to evaporate the solvent and to increase the density of the film. After that the mask was put in close contact with the wafer in a contact-aligner and the photoresist was exposed to near UV light $(350-400 \mathrm{~nm})$ followed by a post-exposure bake. These last steps are made to selectively cross-link the exposed parts of the wafer. The wafer was finally developed in MicroChem's SU-8 Developer.

\section{In a chemistry laboratory using a laser printer}

The method described above can be used to produce structures with a resolution down to the sub- $\mu \mathrm{m}$ range. In the present application, however, most structures are of the order of $20 \mu \mathrm{m}$. A structure of sufficient quality can be produced using a standard laser printer and

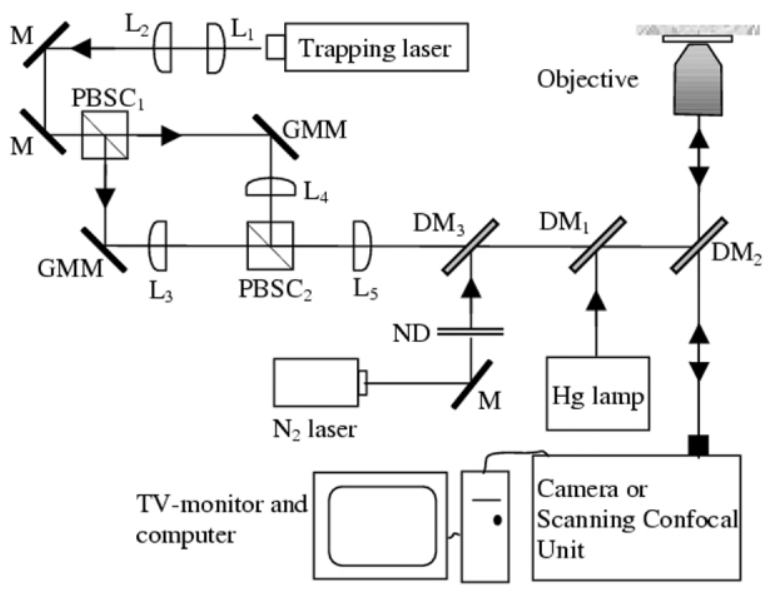

Fig. 1 Schematic representation of the optical setup used in this study. The system consists of a diode pumped $\mathrm{Nd}: \mathrm{YVO}_{4}$ laser and an $\mathrm{Ar}^{+}$pumped titanium-sapphire laser for the optical trapping. A pulsed $\mathrm{N}_{2}$ laser is used for the laser scalpel and an epi-fluorescence and scanning confocal microscope for imaging. A series of dichroic mirrors allow the excitation light from the confocal unit or $\mathrm{Hg}$ lamp and the fluorescence from the sample to pass through, while the light forming both the optical tweezers and the laser scalpel is reflected through the objective. $\mathrm{L}_{1}$ to $\mathrm{L}_{5}$, lenses; $\mathrm{PBSC}_{1}$ to $\mathrm{PBSC}_{2}$, polarizing beam splitting cubes; GMM, Gimbal mounted mirrors; ND, Neutral density filters; $\mathrm{DM}_{1}$ to $\mathrm{DM}_{3}$, dichroic mirrors; $\mathrm{M}$, mirrors. 
utensils available in a reasonably-equipped chemistry laboratory. Similar methods have been shown previously by Tan et al. ${ }^{32}$ Many of our prototypes have been produced in this way, and below there follows a short description of the method. All the work was performed in an ordinary fume cupboard illuminated with a yellow lamp used in darkrooms. The pattern layout, generated in a PC, was printed as negative on standard overhead film using a printer with a resolution of $1200 \mathrm{dpi}$. The wafer was replaced with an ordinary microscope slide that had been cleaned with acetone. The photoresist was spin-coated using the same method as described above. The soft- and post-exposure bake was performed either in a convection oven $\left(T=95^{\circ} \mathrm{C}\right)$ or on a hot plate. The exposure was conducted with an ordinary UV-lamp, where the transparencies were pressed against the coated microscope slide using a silicon glass plate.

\section{Molding}

A 10:1 mixture of PDMS prepolymer and curing agent was mixed carefully and air bubbles in the mixture were removed in an evacuated chamber. The PDMS was thereafter dispensed on the master and dried in a convection oven at $95{ }^{\circ} \mathrm{C}$ for $30 \mathrm{~min}$. After cooling down the PDMS was removed from the master. Next, holes were punched in the PDMS at the position for the reservoirs. The structured PDMS was finally pressed towards a cover glass that had been cleaned with cleaning agent in an ultrasonic bath followed by distilled water rinse. The PDMS and cover glass were then left under pressure for $6 \mathrm{~h}$ to achieve good sealing. Thereafter, the channel system was filled with the liquid media by injection into the source reservoir with a syringe while a vacuum pump was connected to the drain reservoir. Finally, a platinum electrode was placed in each reservoir.

\section{Results}

\section{Changing the media surrounding a cell}

As a first application, we show how cells can be moved between different media. The cells under observation can be investigated and treated without being removed from the field of view of the microscope. This is done either by moving the trapped cell by the motorized gimbal-mounted mirrors within the field of view of the microscope, (typically $50 \mu \mathrm{m}$ ), or by moving the whole channel system by the motorized microscope stage, (typically in the $\mu \mathrm{m}$ to $\mathrm{mm}$ range). The channel system for this experiment is shown in Fig. 2. Three reservoirs containing different media and one test chamber for cell storage were manufactured. First, the channel and reservoir system were filled with buffer (M9). Second, three source reservoirs were filled with equal amounts of solutions. The first source reservoir was filled with a solution of $E$. coli bacteria and buffer. The E. coli used in this work (MC4100 strain) lack flagella

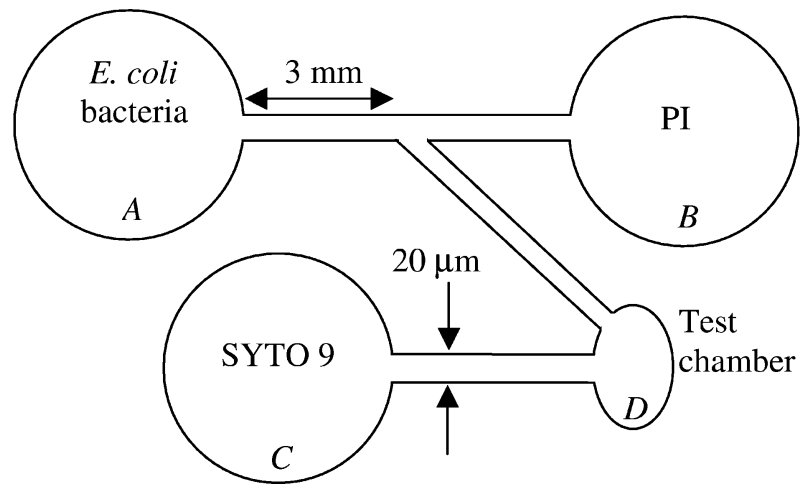

Fig. 2 The figure shows the layout for a system where bacteria are exposed to different media and then placed in the test chamber. Reservoir A was filled with a solution of E. coli and buffer and reservoir B was filled with a solution containing the red fluorescent marker propidium iodide (PI). Reservoir C was filled with SYTO 9. This dye is a green fluorescent nucleic acid stain. which would otherwise increase the movement of the bacteria. The second source was filled with a solution containing the fluorescent marker propidium iodide (PI). This red fluorescent nucleic acid stain does not fluoresce in aqueous solution, but shows a strong emission when attached to the nucleic acids of a bacterium. PI cannot enter bacteria that have intact cell membranes and thus only stains bacteria that have damaged membranes. The third reservoir was filled with SYTO 9. This dye is a green fluorescent stain that can penetrate intact cell membranes and attach to nucleic acids and therefore color all bacteria. Fig. 3 shows a time sequence where bacteria are moved along different paths from the reservoir containing E. coli into the test chamber. Totally four bacteria, indicated by the Roman numerals (I-IV), were moved into the test chamber (Fig. 3D) as follows. First, bacterium (I) was trapped and moved to the test chamber, see (I) in Fig. 3D. Bacterium (I) was fixed to the glass surface when pressed to the coverslip for approximately $5 \mathrm{~s}$ using the tweezers. Second, the two bacteria (II) and (III) were trapped in the E. coli reservoir using the dual trap optical tweezers. They were moved to the reservoir containing PI (Fig. 3B1). Bacterium (II) was moved to the focus of the laser scalpel. After typically five pulses with an energy of $0.6 \mu \mathrm{J}$ the membrane of the cell was damaged and bacterium (II) started to fluorescence in red (Fig. 3B2). This indicates that the cell membrane was broken and the PI was diffusing into the cell staining the nucleic acid. Bacterium (III) was unaffected. Third, the two bacteria (II) and (III) were moved to the test chamber and fixed near bacterium (I), see Fig. 3D. Fourth, bacterium (IV) was picked up from the reservoir containing $E$. coli and moved to the reservoir containing SYTO 9 (Fig. 3C1). After approximately 10 min the SYTO 9 dye had penetrated the intact membrane of bacterium (IV) and started to fluoresce in green (Fig. 3C2). Bacterium (IV) was then moved to the test chamber and fixed to the glass surface near the other bacteria. Fig. 3D shows all the four bacteria (I-IV) in the test chamber fixed to the glass surface. A bacterium looks different when attached to a surface as compared to when it is trapped with the optical tweezers in the bulk solution, due to the optical forces. Thus, the long axis of the trapped bacterium is aligned along the direction of the trapping laser. Fig. 3D shows that no SYTO 9 has diffused through the system since bacterium (I) is not green. Therefore, we conclude that by using optical tweezers in combination with a channel system it is possible to move a cell to a place with a different environment and back again without any influence

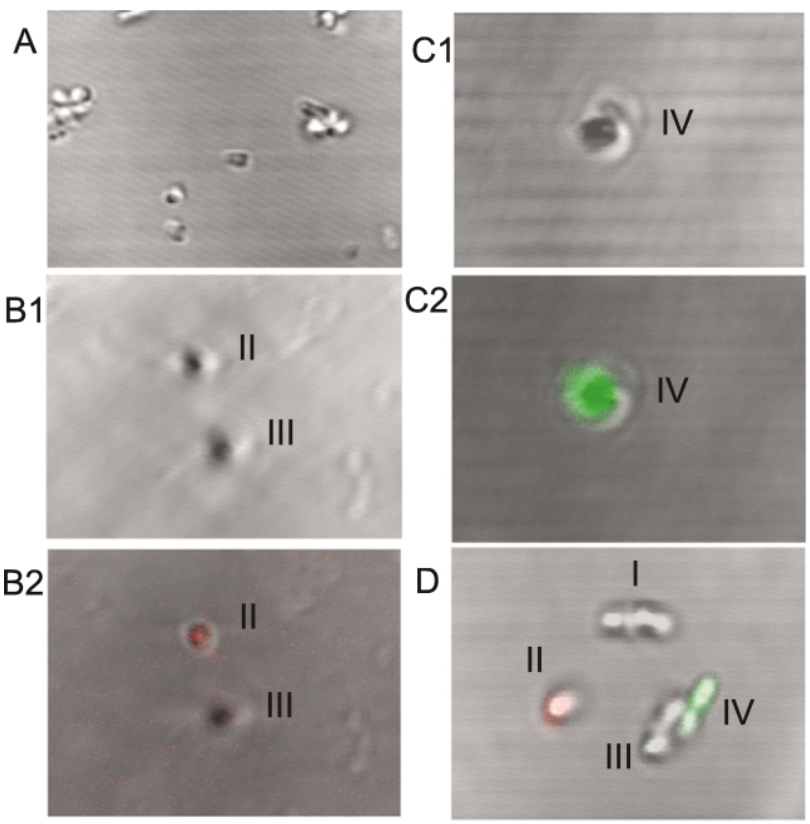

Fig. 3 The figure shows a time sequence where the four bacteria (I-IV) are moved into a test chamber through reservoirs containing different fluorescent dyes. Details of the procedure can be found in the text. 
of contamination from the different channels. This shows that by moving a cell from one media to another with optical tweezers only a very small amount of media, if at all, is being dragged along with the trapped particle. The cells can be left in the chamber, and they can be moved away later for experiments in different environments. In the latter case the surfaces in the reservoir should be coated with a substance that prevents adhesion.

\section{Sorting}

As a second application we show how this technique can be used as a cell sorter. Fig. 4A shows the general layout of this sorter. The system contains three channels, which we have labeled source, drain and collection. Fig. 4B and $\mathrm{C}$ show photographs of such systems produced with the e-beam and laser printer technique, respectively. The channels produced with an e-beam, shown in Fig. $4 \mathrm{~B}$, had a width and a depth of $10 \mu \mathrm{m}$, respectively. The dimensions of the channels produced with a laser printer, Fig. 4C, were typically $20-30 \mu \mathrm{m}$ wide and $15 \mu \mathrm{m}$ deep. A flow of the media in the channels was produced by applying a voltage between the electrodes in the source and drain reservoirs. The flow rate and direction could easily be controlled by varying the voltage between the electrodes. In the present case, with a channel length of $20 \mathrm{~mm}$ and a channel width of $10 \mu \mathrm{m}$, a potential difference of $50 \mathrm{~V}$ gave a flow of approximately $0.2 \mathrm{~mm} \mathrm{~s}^{-1}$. This flow makes a particle pass the field of view of the microscope in approximately $0.5 \mathrm{~s}$. A small flow from the collection reservoir was achieved by applying a potential of $8 \mathrm{~V}$ with respect to the source reservoir. A solution consisting of a 1/1000 mixture of yeast cells and $E$. coli bacteria were introduced in the source reservoirs with M9 as a buffer. Fig. 5 shows microscope images from the region around the junction in the system. The time difference between the first and the last frame is of the order of a minute. When a yeast cell was detected in the source channel (Fig. 5A) the optical tweezers were switched on and directed towards the cell, which was trapped promptly (see Fig. 5B). The trapped particle was moved by the optical tweezers, by changing the direction of the laser beam, into the collection channel, as shown in Fig. 5C to E. The selected cell was now transported by the optical tweezers via the collection channel into the collection reservoir, where it could be extracted using a micromanipulator (not shown).

\section{Discussion and conclusion}

In this report we demonstrate methods to combine optical tweezers with a microfluidic system. In particular, we show how cells can be moved between different reservoirs where they are exposed to different media, without the media being dragged along with the trapped cells. We show how different microfluidic systems can be custom-designed for various types of experiments where the time to develop a new system, from idea to real experiment, can be as short as two days, in particular if the simple method using a laser printer can be used. In this study we used epi-fluorescence and confocal microscopy, but the microfluidic systems can be equally well combined with, for instance, multiphoton microscopy, Raman microscopy or other advanced imaging techniques.

The technique of moving cells between different environments, exemplified in this study by the experiment where a bacterium was moved into a channel with a fluorescent dye, can be generalized to in principle any type of environment. The cells can be moved to regions of the chip with different temperatures, $\mathrm{pH}$ levels, buffer solutions or reservoirs containing nutrition or drugs. This means that we can set up a system where a single cell can be object to many investigations and treatments without being removed from the field of view of the microscope and subsequent dynamics can be studied in real time. The techniques described above can then conveniently be applied for further tests and treatments of the cells under investigation.

The cell sorter that we have demonstrated has some unique features. For instance, the cells to be sorted do not have to pass through the system for identification one by one. The optical tweezers can be directed to pick out a single cell in a medium with a high density of cells. This means that we can use fairly wide channels where a large number of cells can be screened simultaneously. The main drawback with the system is that it will take at least a few seconds to identify, trap and collect one single cell. This type of cell sorter is therefore preferable when a small fraction of cells need to be selected from a large population. It can, in particular, be

A.

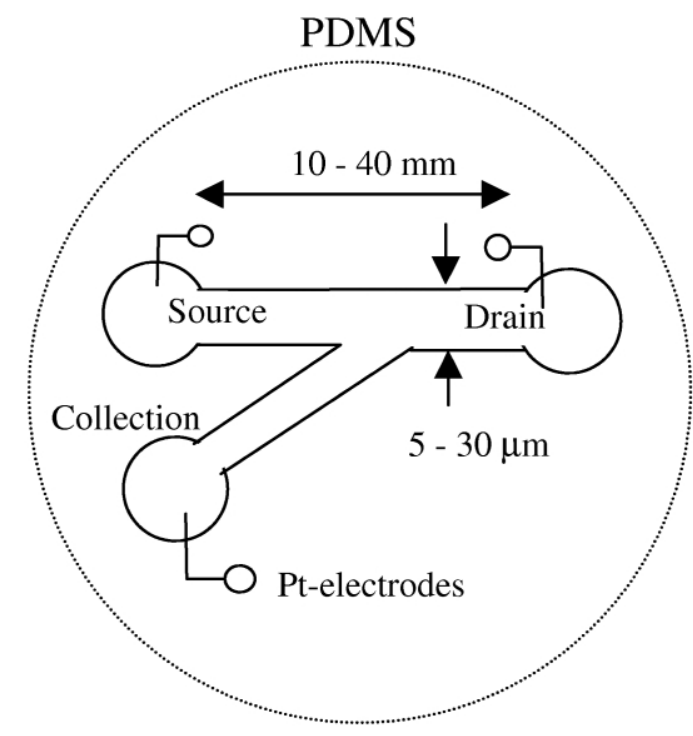

B.

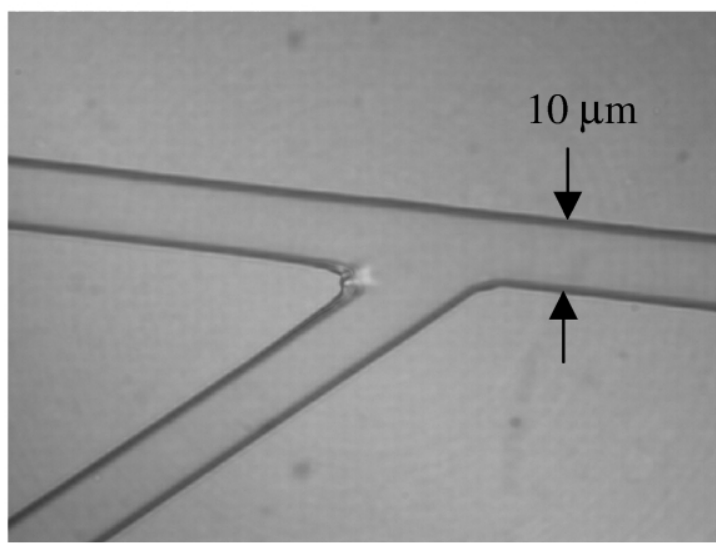

C.

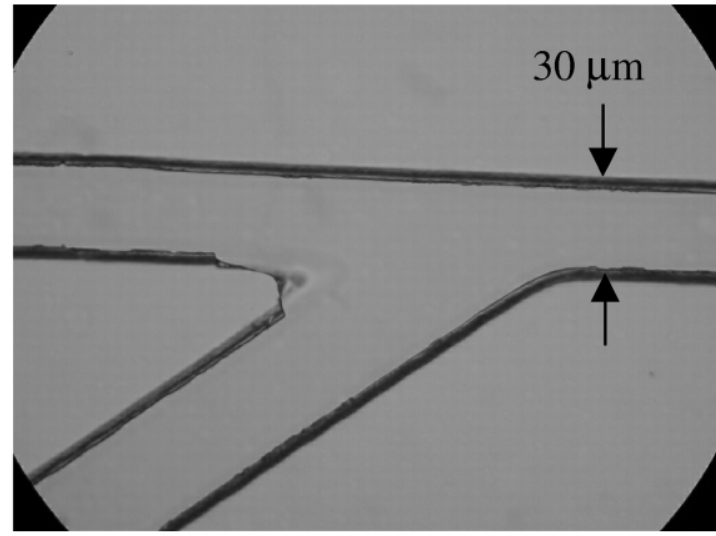

Fig. 4 (A) A schematic of the channel system used for cell sorting. The particles to be sorted are placed in the source reservoir. By applying a voltage between the source and the drain reservoirs using the Pt electrodes, the particles move through the channel. (B) A picture of the channel system produced with an electron beam. (C) A picture of the channel system produced with a laser printer. 

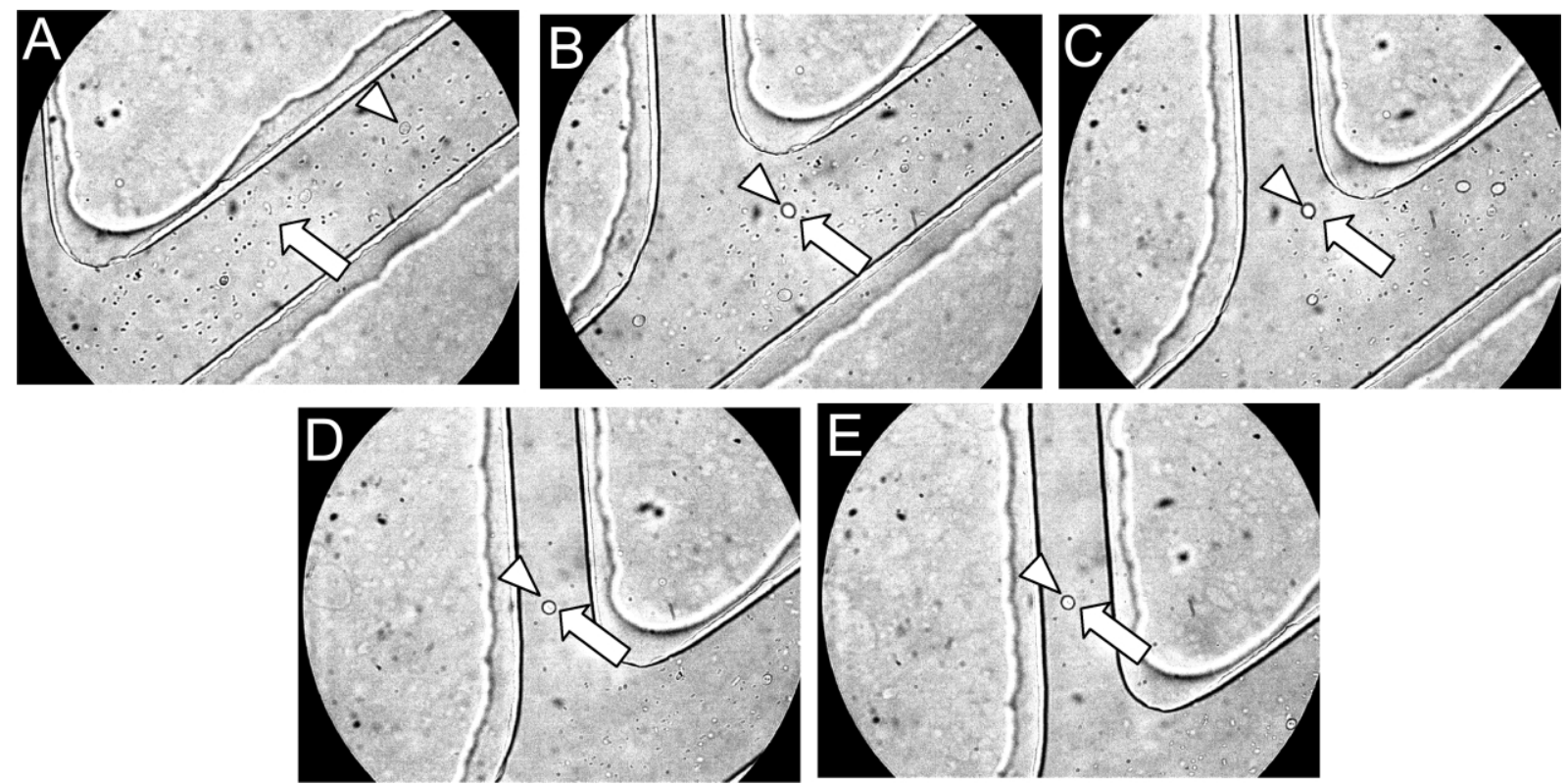

Fig. 5 The figure shows five video frames from a sequence when a yeast cell is trapped with the optical tweezers and moved into the collection channel, whereas the other cells continue straight forward into the drain channel. The arrow indicates the position of the tweezers, and the triangle marks the selected cell.

used if advanced imaging techniques is needed to distinguish the cells to be selected from the culture.

To conclude we have shown that our approach to combine optical tweezers with a microfluidic system, where different cells under investigation can be exposed to different media during the course of an experiment, is a very versatile method that can be used in many types of experiments. We are presently preparing for an experiment where our system will be used to study the oxygenation cycle of hemoglobin. Single optically-trapped red blood cells will be exposed to media that differ in oxygen levels. The oxygenation state of the cells will then be probed by means of Raman spectroscopy.

\section{Acknowledgements}

This research was supported by the Swedish Science Foundation and by SWEGENE through its Center for Biophysical Imaging at Chalmers. We would like to acknowledge the support from the MC2 process lab at Chalmers for valuable support during the production of the channel systems.

\section{References}

1 A. Ashkin, J. M. Dziedzic, J. E. Bjorkholm and S. Chu, Opt. Lett., 1986, 11, 288-290.

2 M. J. Lang and S. M. Block, Am. J. Phys., 2003, 71, 201-215.

3 A. Ashkin, J. M. Dziedzic and T. Yamane, Nature, 1987, 330, 769-771.

4 K. Svoboda and S. M. Block, Annu. Rev. Biophys. Biomolec. Struct., 1994, 23, 247-285.

5 J. Conia, B. S. Edwards and S. Voelkel, J. Clin. Lab. Anal., 1997, 11, 28-38.

6 A. Ashkin and J. M. Dziedzic, Science, 1987, 235, 1517-1520.

7 A. Ashkin and J. M. Dziedzic, Proc. Natl. Acad. Sci. U. S. A., 1989, 86, 7914-7918.

8 A. Ashkin, K. Schutze, J. M. Dziedzic, U. Euteneuer and M. Schliwa, Nature, 1990, 348, 346-348.

9 K. O. Greulich and G. Pilarczyk, Cell Mol. Biol., 1998, 44, 701-710.
10 T. N. Buican, M. J. Smyth, H. A. Crissman, G. C. Salzman, C. C. Stewart and J. C. Martin, Appl. Opt., 1987, 26, 5311-5316.

11 M. Ericsson, D. Hanstorp, P. Hagberg, J. Enger and T. Nyström, J. Bacteriol., 2000, 182, 5551-5555.

12 S. C. Grover, A. G. Skirtach, R. C. Gauthier and C. P. Grover, J. Biomed. Opt., 2001, 6, 14-22.

13 D. C. Duffy, J. C. McDonald, O. J. A. Schueller and G. M. Whitesides, Anal. Chem., 1998, 70, 4974-4984.

14 D. T. Chiu, N. L. Jeon, S. Huang, R. S. Kane, C. J. Wargo, I. S. Choi, D. E. Ingber and G. M. Whitesides, Proc. Natl. Acad. Sci. U. S. A., 2000, 97, 2408-2413.

15 J. R. Anderson, D. T. Chiu, R. J. Jackman, O. Cherniavskaya, J. C. McDonald, H. K. Wu, S. H. Whitesides and G. M. Whitesides, Anal. Chem., 2000, 72, 3158-3164.

16 A. Orfao and A. RuizArguelles, Clin. Biochem., 1996, 29, 5-9.

17 J. M. Ramsey, Nature Biotechnol., 1999, 17, 1061-1062.

18 G. Fuhr and S. G. Shirley, J. Micromech. Microeng., 1995, 5, 77-85.

19 Anne R. Kopf-Sill, Lab Chip, 2002, 2, 42N-47N.

20 D. C. Duffy, O. J. A. Schueller, S. T. Brittain and G. M. Whitesides, J. Micromech. Microeng., 1999, 9, 211-217.

21 T. Yamamoto, T. Nojima and T. Fujii, Lab Chip, 2002, 2, 197-202.

22 M. G. Berrill, J. S. McKenzie and C. Clark, J. Micromech. Microeng., 2000, 10, 56-63.

23 S. Umehara, Y. Wakamoto, I. Inoue and K. Yasuda, Biochem. Biophys. Res. Commun., 2003, 305, 534-540.

24 I. Inoue, Y. Wakamoto, H. Moriguchi, K. Okano and K. Yasuda, Lab Chip, 2001, 1, 50-55.

25 A. Wolff, I. R. Perch-Nielsen, U. D. Larsen, P. Friis, G. Goranovic, C. R. Poulsen, J. P. Kutter and P. Telleman, Lab Chip, 2003, 3, 22-27.

26 A. Y. Fu, C. Spence, A. Scherer, F. H. Arnold and S. R. Quake, Nature Biotechnol., 1999, 17, 1109-1111.

27 J. Krüger, K. Singh, A. O’Neill, C. Jackson, A. Morrison and P. O'Brien, J. Micromech. Microeng., 2002, 12, 486-494.

28 M. Goksör, A. Diez, J. Enger, D. Hanstorp and T. Nyström, EMBO Rep., 2003, 4, 867-871.

29 M. Goksör, J. Enger, K. Ramser and D. Hanstorp, Proc. SPIE, Microarrays and Comb. Techn. For Bio. Appl: Design, Fabrication and Analysis, ed. D. V. Nicolau and R. Raghavachari, 2003, 4966, pp. 50-57.

30 E. Fällman and O. Axner, Appl. Opt., 1997, 36, 2107-2113.

31 K. C. Neuman, E. H. Chadd, G. F. Liou, K. Bergman and S. M. Block, Biophys. J., 1999, 77, 2856-2863.

32 A. Tan, K. Rodgers, J. P. Murrihy, C. O'Mathuna and J. D. Glennon, Lab Chip, 2001, 1, 7-9. 